\title{
Activation of the Kallikrein-Kinin System in Human Plasma by a Serine Protease from Mites
}

\author{
Shoichi Kohmoto, ${ }^{1}$ Yoh Kodera, ${ }^{2}$ Katsunobu TaKahashi, ${ }^{3}$ \\ Hiroyuki Nishimura, ${ }^{4}$ Ayako Matsushima, ${ }^{2}$ \\ and Yuji INADA ${ }^{2, *}$ \\ ${ }^{1}$ Earth Chemical Co., Ltd., Aioi, Hyogo 678-01, Japan \\ ${ }^{2}$ Department of Materials Science and Technology, Toin University of \\ Yokohama, Yokohama 227, Japan \\ ${ }^{3}$ Department of Life Science, Tokyo Institute of Technology, Yokohama 227, Japan \\ ${ }^{4}$ Department of Pathology, Juntendo University School of Medicine, \\ Bunkyo-ku, Tokyo 113, Japan
}

(Received October 26, 1990)

\begin{abstract}
Summary A serine protease was earlier purified to homogeneity from a culture extract of mites and its possible involvement in the allergic reaction was proposed [K. Takahashi et al. (1990): Int. Arch. Allergy Appl. Immun., 91, 80-85]. The serine protease thus obtained was found in the present study to be trypsin-like protease and had high substrate specificity towards the synthetic substrate for blood coagulation factor XIIa. The enzyme gave rise to rhythmic contraction of rat uterine horns in factor XII-deficient human plasma, but not in prekallikrein-deficient plasma. These findings lead us to conclude that this enzyme activates the kallikrein-kinin system in plasma through hydrolysis of prekallikrein in the plasma.
\end{abstract}

Key Words: $\quad$ protease, Dermatophagoides farinae, mite, kallikrein-kinin system, blood coagulation factor XII

It is well known that house dust is one of the major sources of allergens that cause extrinsic asthma or rhinitis. Two species of mites, Dermatophagoides farinae (Df) and Dermatophagoides pteronyssinus (Dp), are recognized as the main source of house dust allergens [1-3]. Chapman and Platts-Mills [4] and Yasueda et al. [5] reported the purification and characterization of the allergenic proteins in extracts made from Df and Dp, and showed them to have molecular weights of 25,000 and $15,000 \mathrm{Da}$, respectively. Recently, proteolytic activity was found in a

*To whom correspondence should be addressed. 
culture extract of Df mites, and the protease responsible was purified to homogeneity and seemed to activate the kallikrein-kinin system in human plasma [6].

Plasma kinins mediate various functions such as vascular permeability enhancement, contraction of smooth muscle, and lowering of blood pressure [7]. In addition, it is noteworthy that kinins are also involved in various allergic reactions $[8,9]$. Bradykinin or kallidin activity is found in nasal secretions of allergenchallenged atopic subjects [10], and kinins stimulate the production of prostaglan$\operatorname{din} \mathrm{E}_{2}$, a chemical mediator of allergic reactions [11].

This paper deals with the activation of the kallikrein-kinin system by Df-protease, and describes the partial amino sequence of the enzyme.

\section{MATERIALS AND METHODS}

The protease used in the present study was purified as described previously from a culture extract of the mite Dermatophagoides farinae [6]. The enzymic activity was 158 units/mg protein when a synthetic substrate of coagulation factor XIIa was used. Bradykinin and synthetic substrates of proteases were purchased from the Peptide Institute, Inc. (Osaka). Human normal plasma and coagulation factor XII-deficient and prekallikrein-deficient plasma were obtained from George King Bio-Medical Inc. (Overland Park, KS). Other chemicals were of analytical grade.

Amino acid analyses (acid hydrolysis) and sequence analyses were carried out in the Toray Research Center (Kamakura) with a Hitachi amino acid analyzer Model 835 (Tokyo) and an amino acid sequencer Model 477A of Applied Biosystems Inc. (Pasadena, CA), respectively. Proteolytic activities were fluorometrically determined at $37^{\circ} \mathrm{C}$ with synthetic substrates [12].

Activation of the kallikrein-kinin system was measured by the contraction of rat uterine horns according to the method of Kato et al. [7] with a minor modification. Briefly, virgin female Wistar rats $(300 \mathrm{~g})$ were injected with $0.37 \mathrm{mg}$ of $\beta$-estradiol (i.p.) $24 \mathrm{~h}$ before experiments. Uterus $(2-3 \mathrm{~cm})$ was vertically mounted in an organ bath containing $10 \mathrm{ml}$ of Munsick buffer $(113 \mathrm{mM} \mathrm{NaCl}, 6$ $\mathrm{mM} \mathrm{KCl}, 0.5 \mathrm{mM} \mathrm{CaCl}_{2}, 1 \mathrm{mM} \mathrm{NaH} \mathrm{PO}_{4}, 30 \mathrm{mM} \mathrm{NaHCO}_{3}$, and $1.6 \mathrm{mM}$ glucose) maintained at $37^{\circ} \mathrm{C}$ and saturated with $95 \% \mathrm{O}_{2}$ and $5 \% \mathrm{CO}_{2}$ under a resting tension of $1 \mathrm{~g}$. After addition of Df-protease (0.06 unit) and various kinds of plasma (25 $\mu 1)$ the contractions were recorded by an isotonic transducer with a recorder (Natsume Seisakusho, Osaka).

\section{RESULTS AND DISCUSSION}

In our previous study, the highly purified Df-protease was found to be a serine protease with a molecular weight of 30,000 ; and its physicochemical properties were clarified [6]. However, the chemical structure and pathophysiological role remained to be clarified. 
The partial amino-terminal amino acid sequence of Df-protease found in the present study, together with those sequences of European hornet chymotrypsin (Vespa crabro protease: VCP II) [13], crayfish trypsin [14], bovine trypsin [15], and bovine chymotrypsin A [16], is shown in Fig. 1. The 12th amino acid residue in Df-protease was not detected. We found, however, that Df-protease in the sequence region between the $\mathrm{N}$-terminus and the 20 th residue was similar to the corresponding region of trypsins or chymotrypsins. Eleven out of a total of 20 $\mathrm{N}$-terminal amino acid residues determined in Df-protease were identical with those of other trypsin-like proteases. Quite recently, similar sequences were reported in proteases Der $f$ III and Der $p$ III from house dust mites (see Fig. 1): four out of 20 [17] and four out of 17 [18] N-terminal amino acid residues, respectively, were different from the sequence of Df-protease obtained in the present study. The amino acid composition of Df-protease was also similar to that of bovine trypsin (data not shown). The substrate specificity of Df-protease was tested with various synthetic substrates of serine-type and other proteases such as blood coagulation factor and fibrinolytic enzyme (Table 1). It is interesting to note that among the synthetic substrates the highest enzymic activity was observed for the synthetic substrate of blood coagulation factor XIIa (Hageman factor). The activity was completely inhibited by phenylmethylsulfonyl fluoride (PMSF), N- $\alpha$-p-tosylL-lysine chloromethyl ketone (TLCK), and soybean and lima been trypsin inhibitors. These results indicate that the Df-protease is a new trypsin-like enzyme with a possibly active serine residue and substrate specificity like that of blood coagulation factor XIIa.

In order to determine if Df-protease could activate the kallikrein-kinin system in human plasma with Df-protease, we carried out the following experiment using rat uterine smooth muscle: Df-protease and normal human plasma were added to Munsick buffer, in which the muscle system was placed, which resulted in rhythmical contraction of the muscle (Fig. 2A). The amount of Df-protease required for the contraction in this system was 0.06 unit (13 pmol), which is comparable to the concentration of XII in plasma $(6-13 \mathrm{pmol})$ [19]. This contraction was enhanced

\begin{tabular}{|c|c|c|c|c|c|c|c|c|c|c|c|c|c|c|c|c|c|c|c|c|}
\hline \multirow[b]{2}{*}{ Df-protease } & \multicolumn{11}{|c|}{1} & \multirow[b]{2}{*}{$*$} & \multirow[b]{2}{*}{$\mathrm{P}$} & \multirow{2}{*}{\multicolumn{2}{|c|}{ Y 6}} & \multirow[b]{2}{*}{ Q } & & \multirow{3}{*}{$\begin{array}{l}\mathrm{L} \\
\mathrm{L}\end{array}$} & \multirow{2}{*}{$\mathrm{Q}$} & \multirow{2}{*}{$\frac{20}{S}$} \\
\hline & I & V & G & G & V & $\mathrm{K}$ & A & $\mathrm{Q}$ & A & G & $\mathrm{D}$ & & & & & & & & & \\
\hline Der $f$ III & I & $\mathrm{V}$ & G & G & $\mathrm{V}$ & $\mathrm{K}$ & A & $\mathrm{L}$ & A & G & $D$ & * & $P$ & $\mathrm{Y}$ & $\mathrm{E}$ & I & $\mathrm{S}$ & & $\mathrm{E}$ & $\mathrm{V}$ \\
\hline Der p III & I & $\mathrm{V}$ & G & S & $E$ & $\mathrm{~K}$ & A & L & A & G & $\mathrm{E}$ & $*$ & $P$ & $\mathrm{Y}$ & $Q$ & I & S & & & \\
\hline Hornet Chymotrypsin (VCP II) & I & $\mathrm{V}$ & G & G & $\mathrm{T}$ & $\mathrm{D}$ & A & $P$ & $\mathrm{R}$ & G & K & $\mathrm{Y}$ & $\mathrm{P}$ & $\mathrm{Y}$ & $\mathrm{Q}$ & $\mathrm{V}$ & S & $\mathrm{L}$ & $\mathrm{R}$ & A \\
\hline Crayfish Trypsin & I & $\mathrm{V}$ & G & G & $\mathrm{T}$ & $\mathrm{D}$ & A & $\mathrm{V}$ & $\mathrm{L}$ & G & $E$ & $\mathrm{~F}$ & $P$ & Y & $\mathrm{Q}$ & $\mathrm{L}$ & S & $\mathrm{F}$ & $Q$ & $\mathrm{E}$ \\
\hline Bovine Trypsin & I & $\mathrm{V}$ & G & G & $\mathrm{Y}$ & $\mathrm{T}$ & C & $G$ & A & $\mathrm{N}$ & $\mathrm{T}$ & $\mathrm{V}$ & $P$ & $\mathrm{Y}$ & $\mathrm{Q}$ & $\mathrm{V}$ & S & $\mathrm{L}$ & $\mathrm{N}$ & S \\
\hline Bovine Chymotrypsin A & I & $\mathrm{V}$ & $\mathrm{N}$ & G & $\mathrm{E}$ & $\mathrm{E}$ & A & $\mathrm{V}$ & $\mathrm{P}$ & $G$ & $\mathrm{~S}$ & $\mathrm{~W}$ & $\mathrm{P}$ & $\mathrm{W}$ & $\mathrm{Q}$ & $\mathrm{V}$ & S & $\mathrm{L}$ & $\mathrm{Q}$ & D \\
\hline
\end{tabular}

Fig. 1. Amino-terminal sequence of Df-protease and other homologous proteases. The amino acid residues that are identical with the corresponding residues of the Df-protease sequence are boxed. * not detected.

Vol. 10, No. 1, 1991 
when we increased the Df-protease concentration. No contraction took place when Df-protease was added without normal plasma or when normal plasma was added without Df-protease. Similar contractions were also observed by addition of Df-protease and coagulation factor XII-deficient human plasma (Fig. 2C). On the other hand, no contraction took place when prekallikrein-deficient human plasma was used (Fig. 2B). As a positive control, the smooth muscle was rhythmically contracted by the addition of $0.5 \mathrm{ng} / \mathrm{ml}$ bradykinin in the same muscle system.

These observations suggest that Df-protease activates the kallikrein-kinin system by its proteolytic action on prekallikrein. This suggestion is compatible with the high specificity of Df-protease towards the synthetic substrate for blood

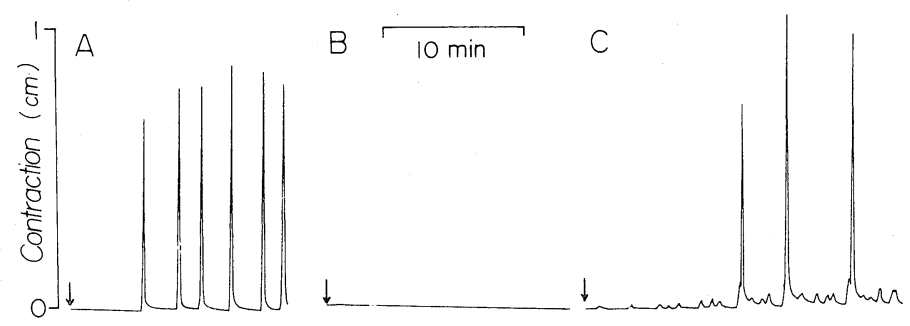

Fig. 2. Contractibility of smooth muscle in rat uterine horns by addition of Df-protease along with various kinds of plasma. A, Normal human plasma $(25 \mu 1)$ plus Df-protease (0.06 unit, 13 pmol); B, Prekallikrein-deficient human plasma $(25 \mu 1)$ plus Df-protease (0.09 unit); C, Factor XII-deficient human plasma (25 $\mu 1)$ plus Df-protease (0.06 unit).

Table 1. Relative enzymic activity of Df-protease towards various synthetic substrates.

\begin{tabular}{llc}
\hline \multicolumn{2}{c}{ Synthetic substrate (for protease) } & Relative activity (\%) \\
\hline Boc-Gln-Gly-Arg-MCA & (Factor XIIa) & $100^{*}$ \\
Boc-Ile-Glu-Gly-Arg-MCA & (Factor Xa) & 73.8 \\
Boc-Val-Leu-Lys-MCA & (Plasmin) & 42.9 \\
Boc-Glu-Lys-Lys-MCA & (Plasmin) & 38.1 \\
Boc-Glu(OBzl)-Ala-Arg-MCA & (Factor XIa) & 26.2 \\
Boc-Val-Pro-Arg-MCA & ( $\alpha$-Thrombin) & 21.4 \\
Pyr-Gly-Arg-MCA & (TPA, Urokinase) & 13.5 \\
Pro-Phe-Arg-MCA & (Tissue kallikrein) & 9.5 \\
Bz-Arg-MCA & (Trypsin) & 4.8 \\
Z-Phe-Arg-MCA & (Plasma kallikrein) & 3.0 \\
Z-Arg-Arg-MCA & (Cathepsin B) & 2.3 \\
Glt-Gly-Arg-MCA & (Urokinase) & 1.2 \\
Arg-MCA & (Cathepsin H) & 0.6 \\
Lys-MCA & & 0.0 \\
Leu-MCA & (Aminopeptidase) & 0.3 \\
Suc-Ala-Ala-Ala-MCA & (Elastase) & 1.1 \\
Gly-Pro-MCA & & 0.0 \\
Lys-Ala-MCA & & 0.3 \\
Pyr-MCA & & 0.0 \\
Suc-Leu-Leu-Val-Tyr-MCA & (Chymotrypsin) & 0.0 \\
\hline
\end{tabular}

*The activity of 158 units/mg protein is expressed as $100 \%$. 
coagulation factor XIIa (Table 1), the enzyme that acts on prekallikrein to form the kallikrein responsible for the proteolysis of high molecular weight kininogen to generate bradykinin.

It is tempting to speculate that Df-protease is involved in the etiology of atopic diseases caused by house dust mites. In fact, some exogenous proteases such as microbial proteases are known to enhance vascular permeability through activation of the kallikrein-kinin system [20]. It is also possible that Df-protease is related to the IgE class-specific immune response against major mite allergens, as kinins are reported to potentiate $\operatorname{IgE}$ production [21].

We are much indebted to Prof. Hiroshi Maeda (Kumamoto University), Prof. Shigehisa Hirose, and Dr. Hiromi Hagiwara (Tokyo Institute of Technology) for valuable discussions.

\section{REFERENCES}

1. Voorhost, R., Spieksma-Boezeman, M.I.A., and Spiksma, F.TH.M. (1964): Is a mite (Dermatophagoides sp.) the producer of the house-dust allergen? Allerg. Asthma, 10, 329-334.

2. Miyamoto, T., Oshima, S., Ishizaki, T., and Sato, S. (1968): Allergic identity between the common floor mite (Dermatophagoides farinae Hughes, 1961) and house dust as a causative antigen in bronchial asthma. J. Allergy, 42, 14-28.

3. Manunsell, K., Wraith, D.G., and Cunnington, A.M. (1968): Mite and house-dust allergy in bronchial asthma. Lancet, i, 1267-1270.

4. Chapman, M.D., and Platts-Mills, T.A.E. (1980): Purification and characterization of the major allergen from Dermatophagoides pteronyssinus-antigen pI. J. Immunol., 125, 587-592.

5. Yasueda, H., Mita, H., Yui, Y., and Shida, T. (1986): Isolation and characterization of two allergens from Dermatophagoides farinae. Int. Arch. Allergy Appl. Immunol., 81, 214-223.

6. Takahashi, K., Aoki, T., Kohmoto, S., Nishimura, H., Kodera, Y., Matsushima, A., and Inada, Y. (1990): Activation of kallikrein-kinin system in human plasma with purified serine protease from Dermatophagoides farinae. Int. Arch. Allergy Appl. Immunol., 91, $80-85$.

7. Kato, H., Nagasawa, S., and Iwanaga, S. (1981): HMW and LMW kininogens, in Methods in Enzymology, Vol. 80, ed. by Lorland, L., Academic Press, New York, pp. 173-175.

8. Proud, D., Naclerio, R.M., Togias, A.G., Kagey-Sobotka, A., Adkinson, N.F., Jr., Norman, P.A., and Lichtenstein, L.M. (1986): Kinins as modifiers of human allergic reactions. $A d v$. Exp. Med. Biol., 198B, 181-187.

9. Proud, D., Baumgarten, C.R., Naclerio, R.M., and Lichtenstein, L.M. (1986): The role of kinins in human allergic disease. N. Engl. Reg. Allergy Proc., 7, 213-218.

10. Proud, D., Togias, A., Naclerio, R.M., 'Crush, S.A., Norman, P.S., and Lichtenstein, L.M. (1983): Kinins are generated in vivo following nasal airway challenge of allergic individuals with allergen. J. Clin. Invest., 72, 1678-1685.

11. Leikauf, G.D., Ueki, I.F., Nadel, J.A., Widdicombe, J.H. (1985): Bradykinin stimulates CI secretion and prostaglandin $\mathrm{E}_{2}$ release by canine tracheal epithelium. Am. J. Physiol., 248, F48-F55.

12. Kawabata, S., Miura, T., Morita, T., Kato, H., Iwanaga, S., Takada, K., Kimura, T., and Sakakibara, S. (1988): Highly sensitive peptide-4-methylcoumaryl-7-amide substrates for blood-clotting proteases and trypsin. Eur. J. Biochem., 172, 17-25.

13. Jany, K.-D., Haug, H. (1983): Amino acid sequence of the chymotryptic protease II from the larvae of the hornet, Vespa crabro. FEBS Lett., 158, 98-102.

14. Titani, K., Sasagawa, T., Woodbury, R.G., Ericsson, L.H., Dorsam, H., Kraemer, M., Neurath, H., and Zwilling, R. (1983): Amino acid sequence of crayfish (Astacus fluviatilis)

Vol. 10, No. 1, 1991 
trypsin $\mathrm{I}_{\mathrm{f}}$. Biochemistry, 22, 1459-1465.

15. Mikes, O., Holeysovsky, V., Tomasek, V., and Sorm, F. (1966): Covalent structure of bovine trypsinogen. The position of the remaining amides. Biochem. Biophys. Res. Commun., 24 , 346-351.

16. Brown, J.R., Hartley, B.S. (1966): Location of disulphide bridges by diagonal paper electrophoresis. The disulphide bridges of bovine chymotrypsinogen A. Biochem. J., 101, 214-228.

17. Hymann, P.W., Chapman, M.D., Aalberse, R.C., Fox, J.W., and Platts-Mills, T.A.E. (1989): Antigenic and structural analysis of group II allergens (Der $f$ II and $\operatorname{Der} p$ II) from house dust mites (Dermatophagoides spp). J. Allergy Clin. Immunol., 83, 1055-1067.

18. Stewart, G.A., Thompson, P.J., and Simpson, R.J. (1989): Protease antigens from house dust mite. Lancet Jul., 15, 154-155.

19. Movat, H.Z. (1979): The plasma kallikrein-kinin system and its interrelationship with other components of blood, in Handbook of Experimental Pharmacology, Suppl. Vol. 25, Bradykinin, Kallidin and Kallikrein, ed. by Erdos, E.G., Springer-Verlag, New York, pp. $1-89$.

20. Molla, A., Yamamoto, T., Akaike, T., Miyoshi, S., and Maeda, H. (1989): Activation of Hageman factor and prekallikrein and generation of kinin by various microbial proteinases. J. Biol. Chem., 264, 10589-10594.

21. Iwata, M., Munoz, J.J., Ishizaka, K. (1983): Modulation of the biologic activities of IgEbinding factor IV. Identification of glycosylation-enhancing factor as kallikrein-like enzyme. J. Immunol., 131,1954-1960. 